\title{
Biosynthesis of selenium nanoparticles from Bacillus species and its applications
}

\author{
B. C. Greeshma \\ Azyme Biosciences Private Limited, Jayanagar, Bangalore-560069 (Karnataka), India \\ M. Mahesh* \\ Azyme Biosciences Private Limited, Jayanagar, Bangalore-560069 (Karnataka), India \\ *Corresponding author. E-mail: mahesh@azymebio.com
}

\begin{abstract}
Selenium nanoparticles have been widely used in the various areas especially in medical field for its anticancer and immune modulatory properties. To reduce the toxic chemicals released by chemical process, the present work was aimed at synthesis of selenium nanoparticles by microbes. In this study, microbes were isolated from soil samples from different areas of Bangalore and screened for the antioxidant activity by DPPH assay. The organism with highest antioxidant activity (IC50 value $=11.6 \mu \mathrm{g} / \mathrm{mL}$ ) was identified as Bacillus species. Under experimental conditions, this microbe along with sodium selenite synthesised selenium nanoparticle indicated by the colour change of the medium to reddish orange. The synthesised selenium nanoparticles were further characterised. From UV-Vis spectrophotometry, the maximum peak was obtained at $266.5 \mathrm{~nm}$. The FT-IR analysis showed peaks at different wavelengths with the maximum of $3200 \mathrm{~cm}^{-1}$ showing the presence of alcoholic group. The shape and size of the selenium nanoparticles was also calibrated by SEM analysis as oval and $209 \mathrm{~nm}-748 \mathrm{~nm}$ respectively. The nanoparticles were further analysed for antimicrobial assays by well diffusion method against $E$. coli, S. aureus, $P$. aeruginosa, St. mutans and antifungal assay against the strains of $C$. albicans, $A$. niger and $A$. flavus. The highest zone of inhibition was observed against E.coli $(1.7 \mathrm{~cm})$ at a concentration of $400 \mu \mathrm{g}$ and maximum for $C$. albicans $(2.5 \mathrm{~cm})$ at a concentration of $400 \mu \mathrm{g}$. Microbial synthesized nanoparticles emerges as a promising medicine in curing different disorders because of its potent antioxidant and antimicrobial activity.
\end{abstract}

Keywords: Antimicrobial, Antioxidant, Inhibition, Selenium nanoparticles, UV-Vis spectrum

\section{INTRODUCTION}

Nanoparticles, the particles with one or more dimensions of the order of $100 \mathrm{~nm}$ or less ( $\mathrm{Li}$ et al.,2011) are being used recently in different fields that has dragged the attention of all the scientists in the field of nanotechnology (Fesharaki et al.,2009; Singh et al.,2014;). The unique properties of nano materials that differ from the bulk metals have considerable application in medicine including cancer therapy, DNA analysis, drug delivery, biosensor and magnetic resonance imaging, apart from agricultural, environmental remediation, pharmaceutical, electronics and commercial industries (Shubharani et al., 2019).

Since the beginning of the life, bacteria have been exposed to metal hence the prokaryotes have the ability to resist heavy metal and metalloid toxicity (Llamosas et al., 2016). Nanoparticles have higher surface area in turn leading to the increase interaction with the bacterial pathogens. Compared to micron particles, these nanoparticles can enter bacterial bodies easily due to their smaller

\section{Article Info}

https://doi.org/

10.31018/jans.v11i4.2188

Received: November 2, 2019

Revised: December 5, 2019

Accepted: December 8, 2019

\section{How to Cite}

Greeshma, B.C. and Mahesh, M. (2019) Biosynthesis of selenium nanoparticles from Bacillus species and its applications. Journal of Applied and Natural Science, 11 (4): $810-815$ https:// doi.org/10.31018/

jans.v11i4.2188 size and exert stronger effects on the targeted bacterial surface (Singh et al., 2014). In recent times, silver, gold and selenium nanoparticles are highly significant.

In medical field, selenium nanoparticle has been marked with high biological activity and low toxicity which is of interest in particular. The different species of selenium have varied distribution in environment based on the prevailing redox conditions. There are various methods like physical, chemical, biological and hybrid methods for the synthesis of nanoparticle (Fesharaki et al., 2009; $\mathrm{Li}$ et al., 2011). Among these, physical and chemical process are more popular but due its toxic nature it limits itself in the biomedical applications. Therefore, in order to increase its application in this field the reliable, non-toxic and eco-friendly method usage is of great importance. One of the methods, to achieve these required criteria is the synthesis of nanoparticles using the microorganisms. The biogenetic enzyme method is the superior way of producing nanoparticle compared to the chemical method which has high production 
rate in short span of time in addition to the definite shape and sized particles but, with the release of toxic chemicals that are harmful for environment as well as humans (Li et al., 2011).

As microbes are diverse in nature they can be usefully exploited for the synthesis and harvesting of nanoparticles (Singh et al., 2014). For example, Li et al., 2011; Wang et al.,2011 have reported the synthesis of nanoparticles (selenium and silver) by species like Capsicum annuum L. extract, Escherichia coli, Bacillus subtilis respectively (Zhang et al., 2011). Depending upon the mechanism involved, there could be two types of synthesis of metallic selenium nanoparticles i.e. intracellular and extracellular. Extracellular accumulation could be directly linked to anaerobic respiration whereas, the intracellular might be the result of selenite detoxification (Llamosas et al., 2016). Selenium turns out to be the essential trace element which is now valued as a chemoprevention compounds. Within a proper dose the selenium acts as a chemotherapeutic agent by inducing cancer cell apoptosis with the minimal side effects on normal cells.

In this research work, the selenium nanoparticles were synthesised by Bacillus species isolated from soil samples collected from various places in Bangalore and their antibacterial and antifungal activities were studied. Furthermore, they were also characterised by UV-analysis, Scanning electron microscopy (SEM) and Fourier transform infrared (FTIR).

\section{MATERIALS AND METHODS}

Isolation and identification of microorganism: Collection of soil samples: The soil sample was gathered from different areas of Bangalore such as Kengeri Satellite Town, Jayanagar, Rajarajeshwarinagar, Rajajinagar and Sahakarnagar under sterile conditions in the month of January 2019 and it was transported to research facility for further examinations.

Isolation and pure culture preparation of microorganisms: Isolation of native microbes from the soil sample was done by pour plate method. In this method, approximately $200 \mu \mathrm{L}$ of soil suspension (1 gram of soil sample mixed with $10 \mathrm{~mL}$ saline) was added to petri plates on top of which Luria Bertani Agar medium (Tryptone - 1g; Yeast extract - 0.6g; Sodium Chloride - 2g; Agar $-2 \mathrm{~g}$; Distilled water $-100 \mathrm{ml}$ ) was poured, allowed to solidify and incubated at $37^{\circ} \mathrm{C}$ for 24 hours. From the refined plates the morphologically non identical strains were confined and developed on a similar medium for unadulterated culture plating ie., Pure culture.

Screening of antioxidant producing microbes: The sterile LB broth (Tryptone-1g; Yeast extract$0.6 \mathrm{~g}$; Sodium Chloride-1g; Distilled water-100ml) was inoculated with all the isolated microorgan- isms separately and incubated at $37^{\circ} \mathrm{C}$. The free radical scavenging activity (DPPH assay) was determined after every 24hours for 4 days using the method given by Blois (Marsden, 1958). The cell free supernatant with different concentrations ranging from $10 \mu \mathrm{l}$ to $50 \mu \mathrm{l}$ was pipetted into different test tubes. $3 \mathrm{ml}$ of freshly prepared DPPH solution (absorbance was pre-set to 1) was added and incubated in dark chamber for 15 minutes. The absorbance was measured at $517 \mathrm{~nm}$ using methanol as the blank. The percentage of inhibition was calculated for each concentration and IC50 value was determined.

Identification of antioxidant producing microbes: Different biochemical tests were performed to identify the isolate which include Indole test, Methyl red test, Voges Proskauer s reaction, Citrate Utilisation, Urease production, Nitrate reduction, $\mathrm{H}_{2} \mathrm{~S}$ production, Triple sugar iron agar test, Gelatine test, Carbohydrate test, Cellulase Agar, Starch Agar, Lipase Agar, Casein Agar, Catalase test and Oxidase test.

Optimisation of physical parameters for production of antioxidants:

Effect of temperature: The LB broth was prepared and inoculated with bacteria, incubated at different temperatures viz. room temperature, $30^{\circ}$ $\mathrm{C}, 35^{\circ} \mathrm{C}, 40^{\circ} \mathrm{C}, 45^{\circ} \mathrm{C}$ for optimum incubation period. The inoculated broth was centrifuged at $6000 \mathrm{rpm}$ for 10 minutes at $4^{\circ} \mathrm{C}$ and the supernatant was collected. This was then examined using the DPPH assay at different time period.

Effect of $\mathrm{pH}$ : The LB broth with different $\mathrm{pH}$ ranging from $\mathrm{pH} 4-\mathrm{pH} 8$ was prepared. The $\mathrm{pH}$ was set using $1 \mathrm{M} \mathrm{HCl}$ and $1 \mathrm{~N} \mathrm{NaOH}$. This broth was then inoculated with bacterial culture, incubated at optimum temperature. Later, the cell free supernatant was suspended for DPPH assay after optimum incubation time.

Biosynthesis of selenium nanoparticles and its characterisation: The synthesis of selenium nanoparticles was done using ascorbic acid and sodium selenite. Firstly, 40mM Ascorbic acid and $30 \mathrm{mM}$ Sodium selenite were prepared and mixed. This was prepared in two conical flasks which were labelled as control and sample. Under sterilized conditions, the conical flask labelled as sample was inoculated with the bacterial culture whereas the conical flask named as control was devoid of organism. The synthesised nanoparticles absorption spectrum was studied using UVVIS spectrophotometer at regular intervals and the absorbance was recorded between 200-800nm. The absorbance at which the peak was obtained was noted down. This incubated cell filtrate was centrifuged at $10000 \mathrm{rpm}$ for 30 minutes, out of which maximum amount of pellet was collected. The obtained pellet was washed using ethanol for few minutes and dried. To collect the selenium nanoparticles, the pellet was re-suspended in 
$0.1 \mathrm{M}$ phosphate buffer saline $(\mathrm{pH} \mathrm{7.4)}$ and centrifuged at $10000 \mathrm{rpm}$ for 20 minutes at $4^{\circ} \mathrm{C}$, later scraped and was poured on to the petri plates. The petri plates were allowed to dry. The dried particles were collected i.e., the selenium nanoparticles was used for further analysis. (Ramamurthy et al., 2013)

Characterization by FTIR and SEM: FT-IR range was acquired utilizing spectrophotometer (Shimadzu-00463 model) in the ghostly locale of $4000-600 \mathrm{~cm}^{-1}$ utilizing a goal of $4 \mathrm{~cm}-1$ and 64 coded sweeps. FT-IR range affirms the surface science and functional groups gathering in charge of adjustment of nanoparticles. A Hitachi (Model SU 3500) ultrahigh resolution field emission scanning electron microscope was used for inspecting the particles morphology, i.e. to determine the physical dimensions, shapes and size distribution of nanoparticles

Applications of selenium nanoparticles:

Antibacterial activity: The impact of SeNPs on the antimicrobial movement of the synthesized nanoparticles was assessed by LB agar well diffusion method against various pathogens such as Escherichia coli, Staphylococcus aureus, Strepto-

Table 1. Results of biochemical tests.

\begin{tabular}{ll}
\hline Tests & Result \\
\hline Indole test & Negative \\
Methyl red test & Positive \\
Voges Proskauer s test & Negative \\
Citrate utilization test & Negative \\
Urease production & Negative \\
H2S production & Negative \\
Carbohydrate test & Positive \\
Triple sugar iron agar test & Positive only for Sucrose \\
Gelatin test & Positive \\
Cellulase Agar & Negative \\
Starch Agar & Positive \\
Lipase Agar & Positive \\
Casein hydrolysis & Negative \\
Catalase test & Positive \\
Oxidase test & Negative \\
Nitrate reduction test & Positive \\
\hline
\end{tabular}

coccus mutans and Pseudomonas aeruginosa. A known amount of suspension of each strain for microbes was swabbed consistently onto sterile LB agar plates utilizing spreader. Wells of $10 \mathrm{~mm}$ width were drilled into the agar medium utilizing plug borer. Utilizing a pipette, $50 \mu \mathrm{l}$ of different gradient sample(100-400 $\mu \mathrm{g})$ was added to the wells. After incubating at $37^{\circ} \mathrm{C}$ for $24 \mathrm{hrs}$, the diverse dimensions of zone of restraint were tabulated.

Antifungal assay: Antifungal activity was evaluated by potato dextrose agar (PDA: Potato-200g; Dextrose-20g; Agar-20g; Distilled water-1000ml) well dissemination strategy. Different sorts of pathogenic microorganisms, Candida albicans, Aspergillus niger and $A$. flavus was swabbed reliably onto sterile PDA plates using a spreader. Wells of $10 \mathrm{~mm}$ width were bored into the agar medium using plug borer. Using a pipette, $50 \mu$ l of various concentrations of nanoparticles $(100-500 \mu \mathrm{g})$ was added to each one of the wells and incubated at $37^{\circ} \mathrm{C}$ for $24 \mathrm{hrs}$, the various elements of zone of limitation were evaluated after the incubation.

Evaluation of antioxidant assay: The antioxidant assays included of both DPPH and ABTS assay. The selenium nanoparticles synthesised was weighed and dissolved in methanol $(10 \mathrm{mg} / \mathrm{mL})$ completely. DPPH assay was performed according to the procedure described by Blois.

Evaluation of antioxidant activity by $A B T S$ method: Different concentrations of sample ranging from $100 \mu \mathrm{g}$ to $500 \mu \mathrm{g}$ were pipetted into different test tubes. $3 \mathrm{ml}$ of ABTS solution (absorbance was pre-set to 1) was added and incubated in dark chamber for 15 minutes. The absorbance was measured at $734 \mathrm{~nm}$ using methanol as the blank. The percentage of inhibition was calculated for each concentration and IC50 value was determined.

\section{RESULTS AND DISCUSSION}

Isolation of microorganism from soil samples: After isolation, about 56 bacterial colonies were

Table 2. Showing the zone of inhibition for antibacterial assay.

\begin{tabular}{|c|c|c|c|c|c|c|c|c|}
\hline \multirow{3}{*}{$\begin{array}{l}\text { Concentration } \\
(\mu \mathrm{l})\end{array}$} & \multicolumn{8}{|c|}{ Zone of inhibition $(\mathrm{cm})$} \\
\hline & \multicolumn{2}{|l|}{ E.coli } & \multicolumn{2}{|c|}{ Sta. aureus } & \multicolumn{2}{|c|}{$\begin{array}{l}\text { Pseudomonas } \\
\text { aeruginosa }\end{array}$} & \multicolumn{2}{|c|}{ Str. mutans } \\
\hline & control & sample & control & sample & control & sample & control & sample \\
\hline 100 & $1.2 \mathrm{~cm}$ & - & $1.2 \mathrm{~cm}$ & $1.0 \mathrm{~cm}$ & $1.2 \mathrm{~cm}$ & $0.9 \mathrm{~cm}$ & - & - \\
\hline 200 & $1.4 \mathrm{~cm}$ & - & $1.3 \mathrm{~cm}$ & $1.1 \mathrm{~cm}$ & $1.5 \mathrm{~cm}$ & $1.4 \mathrm{~cm}$ & $1.4 \mathrm{~cm}$ & - \\
\hline 300 & $1.4 \mathrm{~cm}$ & - & $1.3 \mathrm{~cm}$ & $1.4 \mathrm{~cm}$ & $1.3 \mathrm{~cm}$ & $1.2 \mathrm{~cm}$ & $1.2 \mathrm{~cm}$ & - \\
\hline 400 & $1.5 \mathrm{~cm}$ & $1.7 \mathrm{~cm}$ & $1.4 \mathrm{~cm}$ & $1.3 \mathrm{~cm}$ & $1.6 \mathrm{~cm}$ & $1.5 \mathrm{~cm}$ & $1.4 \mathrm{~cm}$ & - \\
\hline
\end{tabular}

Table 3. Showing the zone of inhibition for antifungal assay.

\begin{tabular}{lllllll}
\hline \multirow{2}{*}{$\begin{array}{l}\text { Concentration } \\
\boldsymbol{\mu l})\end{array}$} & \multicolumn{5}{c}{ Zone of inhibition (cm) } \\
\cline { 2 - 7 } & \multicolumn{2}{l}{ Candida albicans } & \multicolumn{2}{l}{ Aspergillus niger } & \multicolumn{2}{l}{ Aspergillus flavus } \\
\cline { 2 - 7 } & Control & Sample & Control & Sample & Control & Sample \\
\hline 100 & $1.1 \mathrm{~cm}$ & $1.7 \mathrm{~cm}$ & $1.0 \mathrm{~cm}$ & $1.5 \mathrm{~cm}$ & $0.9 \mathrm{~cm}$ & $1.2 \mathrm{~cm}$ \\
200 & $1.3 \mathrm{~cm}$ & $2.1 \mathrm{~cm}$ & $1.1 \mathrm{~cm}$ & $1.8 \mathrm{~cm}$ & $1.0 \mathrm{~cm}$ & $1.3 \mathrm{~cm}$ \\
300 & $1.2 \mathrm{~cm}$ & $2.1 \mathrm{~cm}$ & $1.2 \mathrm{~cm}$ & $1.9 \mathrm{~cm}$ & $1.1 \mathrm{~cm}$ & $1.5 \mathrm{~cm}$ \\
400 & $2.1 \mathrm{~cm}$ & $2.5 \mathrm{~cm}$ & $1.5 \mathrm{~cm}$ & $2.0 \mathrm{~cm}$ & $1.2 \mathrm{~cm}$ & $1.6 \mathrm{~cm}$ \\
\hline \hline
\end{tabular}


Table 4. Showing the percentage inhibition for antioxidant assay.

\begin{tabular}{lll}
\hline \multirow{2}{*}{$\begin{array}{l}\text { Amount of } \\
\text { sample }(\boldsymbol{\mu g})\end{array}$} & \multicolumn{2}{c}{ Percentage inhibition } \\
\cline { 2 - 3 } & Control & Sample \\
\hline 100 & 7.6 & 9.7 \\
200 & 7.9 & 13.6 \\
300 & 8.7 & 16.3 \\
400 & 10.5 & 18.9 \\
500 & 10.7 & 24.5 \\
\hline
\end{tabular}

Screening

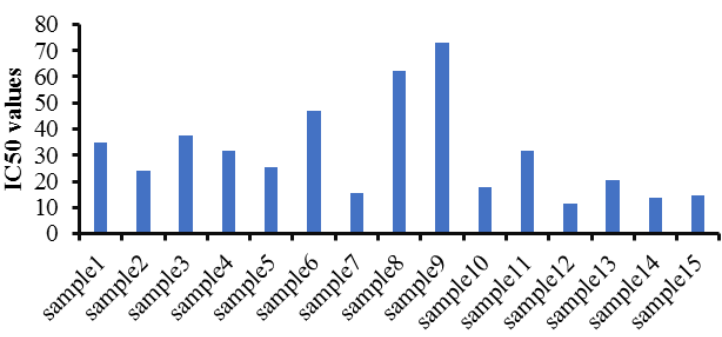

Fig.1. Graph showing IC50 values of different organisms collected from soil sample of Kengeri Satellite Town, Jayanagar, Rajarajeshwarinagar, Rajajinagar and Sahakarnagar.

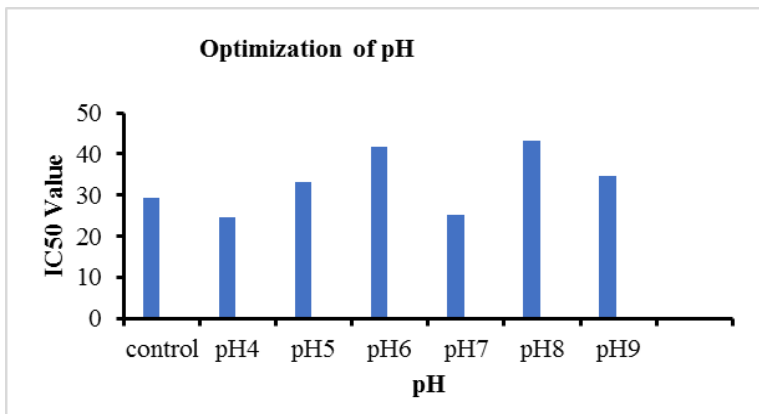

Fig. 2. Graph showing IC50 values at different $\mathrm{pH}$.

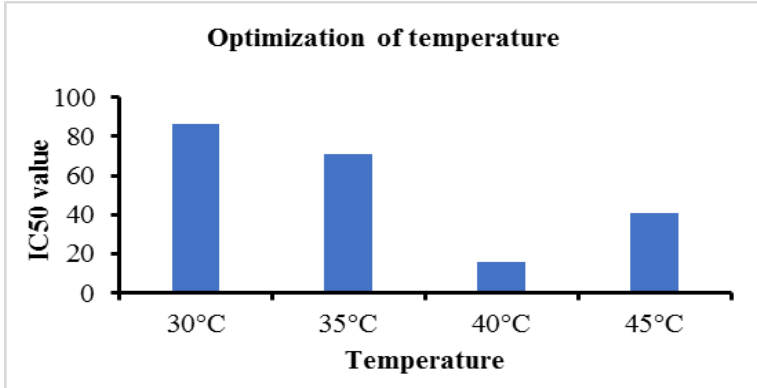

Fig. 3. Graph showing IC50 values at different temperature.

observed out of which 15 bacterial colonies were selected based on their distinct morphological features and used for further works.

Screening of antioxidant producing microbes: DPPH analysis was carried on for all the selected 15 microorganisms. Based on the percentage inhibition and IC50 values, organism labelled as 12 was selected for the biochemical analysis since it showed less IC50 value $(11.6 \mu \mathrm{g} / \mathrm{mL})$ as

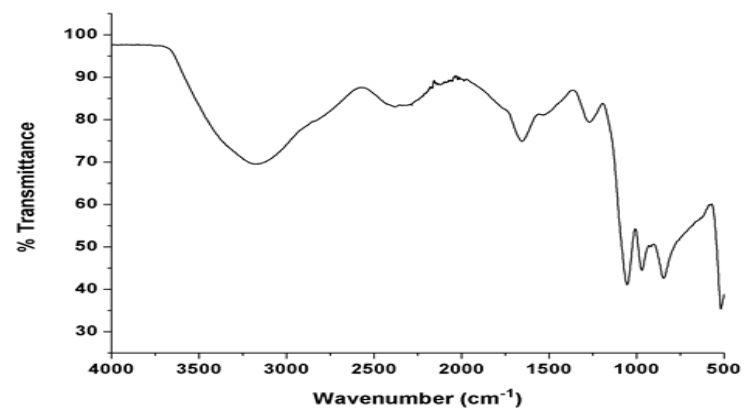

Fig. 4. Graph showing FTIR spectra.

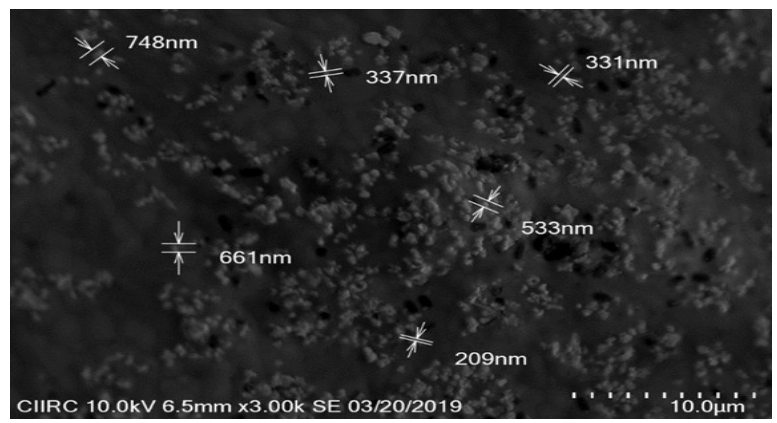

Fig. 5. Image showing the particle size of selenium nanoparticles.

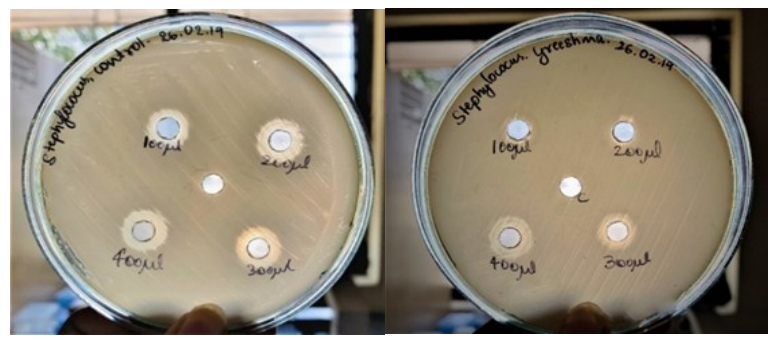

Fig. 6. Zone of inhibition against Sta. aureus.

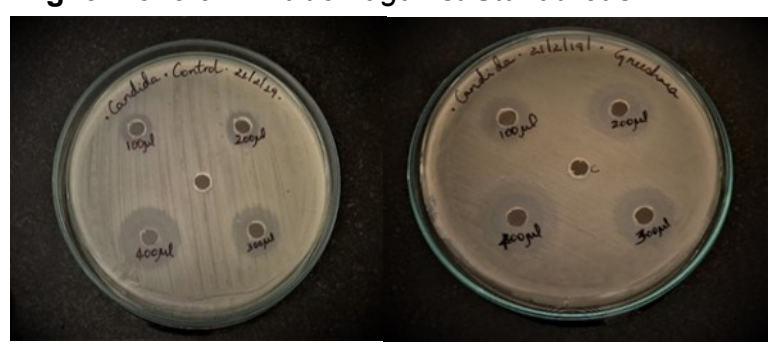

Fig. 7. Antifungal assay of control and sample.

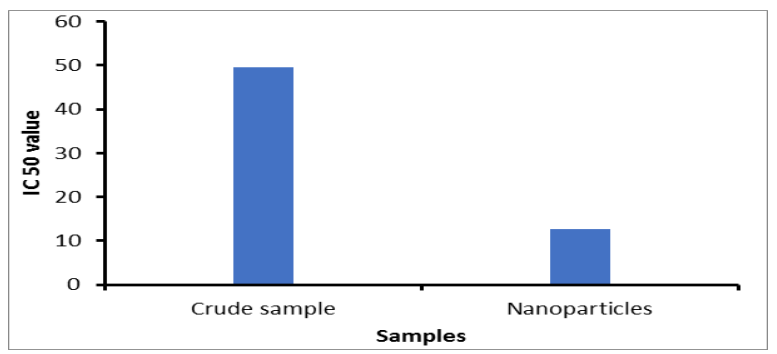

Fig. 8. Graph showing IC50 value of crude sample and nanoparticles.

shown in Figure 1.

Identification of antioxidant producing microbes: The organism isolated from the soil 
appeared as a smooth, translucent white province. It was distinguished as Gram positive rods from Gram staining. From the biochemical tests, the bacterium was distinguished as Bacillus species since it indicated positive outcome for sugar fermentation, catalase, starch hydrolysis and methyl red tests. The obtained results for the tests are summarised in Table 1.

\section{Optimisation of physical parameters for} production of antioxidants

Effect of $\mathrm{pH}$ : The $\mathrm{pH}$ plays an important role in the production of antioxidant. Production of antioxidant was carried out at different $\mathrm{pH}$ viz. $\mathrm{pH} 4$, $\mathrm{pH} 5, \mathrm{pH} 6, \mathrm{pH} 7$ and $\mathrm{pH} 8$. The highest production of antioxidant was observed at $\mathrm{pH} 7$ with an IC50 value of $24.34 \mathrm{mg}$ obtained from the graph shown in Figure 2. Similar results were observed with (Mahesh et al., 2013) where the highest production of ALP (3.4 to $4.1 \mathrm{U} / \mathrm{mg}$ ) was observed at $\mathrm{pH}$ 7- $\mathrm{pH} 8$.

Effect of temperature: The temperature plays a significant role in the antioxidant production. The production of antioxidant was carried out at varied temperature like room temperature, $30^{\circ} \mathrm{C}, 35^{\circ} \mathrm{C}$, $40^{\circ} \mathrm{C}, 45^{\circ} \mathrm{C}$. The highest antioxidant production was observed at temperature $40^{\circ} \mathrm{C}$ with an IC50 of 15.78 obtained from the graph (Figure 3 ) is in accordance with (Mahesh et al., 2013) during the production of ALP where the maximum production was noted to be 3.5 to $4.2 \mathrm{U} / \mathrm{mg}$ protein between $35^{\circ} \mathrm{C}-40^{\circ} \mathrm{C}$.

Biosynthesis of selenium nanoparticles: The reduction of selenium ions into Selenium nanoparticles induced by bacteria was evident by the change in colour from light brown to reddish orange, which is the most significant property of nanoparticles. After the addition of acidic sodium selenite solution, the colour changed to orange within 5hours of incubation indicating the formation of selenium nanoparticles. The results obtained were similar to the results observed by (Zhang et al., 2011) where the colour of media changed from greyish liquid to red with the 6hours of incubation.

Characterisation of selenium nanoparticles: The size, structure and shape of nanoparticles plays an important role in the determination of particles property. There are numerous methods to characterise the synthesised of nanoparticles such as UV-visible spectrophotometry, Fourier transform infrared and Scanning Electron Microscopy are widely used.

UV-vis spectrum analysis: The UV-vis spectrum of biosynthesised selenium nanoparticles from bacteria is shown in figure below. The absorbance spectrum was measured in the range of 200$800 \mathrm{~nm}$ and the absorption peak was observed with maxima at $266.5 \mathrm{~nm}$, indicating the formation of selenium nanoparticles in the samples. The above result is in accordance with (Ali et al., 2013) where UV-vis spectra of nanoparticles at 540nm was observed.

FT-IR analysis: FT-IR spectra of the selenium nanoparticles are shown in Figure 4 and the results presented showed the peak value around $800 \mathrm{~cm}^{-1}$ may be due to the presence alkyl chloride. The absorption peak around $950 \mathrm{~cm}^{-1}$ can be the peak of alkene. The peak at $1050 \mathrm{~cm}^{-1}$ represents the alcoholic group. The peak at 1250 and $1600 \mathrm{~cm}^{-1}$ might be due to the presence of amine. The peak at $3200 \mathrm{~cm}^{-1}$ is due to the alcoholic group. Spectra confirmed the presence of different functional groups, may be responsible for reduction and stabilizationof selenium nanoparticles. The present work is in accordance with ( Zhang et al., 2011)

SEM analysis: The SEM results showed that the biosynthesised selenium nanoparticles by bacteria were oval in shape and the size ranged from $209 \mathrm{~nm}-748 \mathrm{~nm}$ (Figure 5) which is typical of the absorption of metallic selenium nanocrystals. This is similar to the obtained results of (Fesharaki et al., 2010) where the particles ranged in size from 100 to $550 \mathrm{~nm}$.

Antimicrobial assay: Antibacterial potential of both sample and control measured according to the zone of inhibition against various bacterial species like E.Coli, Sta. aureus, Streptococcus mutans, Bacillus cereus and $P$. aeruginosa showed that the antibacterial activity was significant in just three strains i.e., Str. mutans, Bacillus cereus and E.coli (Table 2). The moderate zone of inhibition was seen in the Str. mutans and Bacillus cereus. The highest zone of inhibition was seen against the E.coli $(1.7 \mathrm{~cm})$ at the concentration of $400 \mu \mathrm{g}$. (Figure 6) which is in accordance with the reported results of (Shahzadi et al., 2018).

Antifungal assay: Antifungal potential of both sample and control was measured according to the zone of inhibition against various fungal species like $C$. albicans, $A$. niger and $A$. flavus by well diffusion method.(Table 3 ) The antifungal activity was moderately seen in all the three strains with the highest against $C$. albicans $(2.5 \mathrm{~cm})$ at the concentration of $400 \mu \mathrm{g}$ (Figure 7 ) which is in accordance with the results of (Mallmann et al., 2015).

Antioxidant assay: The synthesised nanoparticles antioxidant activity showed no significant result with DPPH assay (Table 4). From the results of ABTS assay (Figure 8), it was observed that the nanoparticles $(\mathrm{IC50}=12.57 \mu \mathrm{g})$ were better free radical scavengers compared to that of the crude sample (IC50 $=49.5 \mu \mathrm{g})$. This result is in accordance with (Salari et al., 2019) where the antioxidant activity of nanoparticles was evident with an IC50 value of $0.70 \pm 0.08 \mathrm{mg}$.

\section{Conclusion}

The SeNPs can be utilized for different applica- 
tions particularly in medication, because of their restorative properties, for example, low toxicity, better reactivity, low measurement, and so on. The synthesis of selenium nanoparticles and their characterisation with bacteria were noticed from UV-vis, Spectra, FT-IR and SEM images. The biosynthesized Selenium nanoparticle showed oval shape with the size range of $209 \mathrm{~nm}-748 \mathrm{~nm}$. The biosynthesized SeNPs could be a powerful cancer prevention agent and antimicrobial operator to treat ailments brought about by grampositive and parasitic strains might be utilized to execute the pathogens for avoiding diseases. Nonetheless, further research is expected to improve the union proficiency, control of molecule size and its application in prescription and human services.

\section{REFERENCES}

1. Ali, E. N., El-Sonbaty, S. M. and Salem, F. M. (2013). Evaluation of selenium nanoparticles as a potential chemopreventive agent against lung carcinoma, International Journal of Pharmaceutical, Biological and Chemical Sciences, 2 (4): 38-46

2. Helga Fernández Llamosas, Laura Castro, María Luisa Blázquez, Eduardo Díaz and Manuel Carmona (2016). Biosynthesis of selenium nanoparticles by Azoarcus sp. Microb Cell Fact (2016) 15:109 DOI 10.1186/s12934-016-0510-y

3. Mahesh, M., Somashekhar, R., Preenon, Bagchi, Puttaiah, E. T. (2015) Optimization for the production of extracellular alkaline phosphatase from Proteus mirabilis. J Bioprocess Biotech, 5: 213 doi: 10.4172/2155-9821.1000213 P

4. Mallmann, E. J., Cunha, F.A., Castro, B.N., Maciel, A.M., Menezes, E.A., Fechine, P.B. (2015). Antifungal activity of silver nanoparticles obtained by green synthesis. Rev Inst Med Trop Sao Paulo. 2015;57 (2):165-167.doi:10.1590/S0036-
46652015000200011

5. Nidhi Singh, Prasenjit Saha, Karthik Rajkumar and Jayanthi Abraham (2014). Biosynthesis of silver and selenium nanoparticles by Bacillus sp. JAPSK2 and evaluation of antimicrobial activity, Der Pharmacia Lettre, 6 (1):175-181

6. Parisa Jafari Fesharaki, Pardis Nazari, Mojtaba Shakibaie, Sassan Rezaie, Maryam Banoee, Mohammad Abdollahi, Ahmad Reza Shahverdi (2010). Biosynthesis of selenium nanoparticles using Klebsiella pneumoniae and their recovery by a simple sterilization process, Brazilian Journal of Microbiology, 41: 461-466

7. Ramamurthy. C. H., Sampath, K.S., Arunkumar, P., Suresh Kumar, M., Sujatha, V. (2013). Green synthesis and characterization of selenium nanoparticles and its augmented cytotoxicity with doxorubicin on cancer cells. Bioprocess Biosyst. Eng., 36: 1131 1139.

8. Shamaila Shahzadi, Nosheen Zafar and Rehana Sharif (2018). Antibacterial activity of metallic nanoparticles, Bacterial Pathogenesis and Antibacterial Control, Sahra, IntechOpen, DOI: 10.5772/ intechopen.72526.

9. Shubharani, R., Mahesh, M. and V. N. Yogananda Murthy (2019). Biosynthesis and characterization, antioxidant and antimicrobial activities of selenium nanoparticles from ethanol extract of Bee Propolis. $J$ Nanomed Nanotechnol.10:1 DOI: 10.4172/21577439.1000522

10.Wenjie Zhang, Zhijuan Chena, Hao Liu, Liang Zhang, Ping Gao, Daping Li (2011). Biosynthesis and structural characteristics of Selenium nanoparticles by Pseudomonas alcaliphila, Colloids And Surfaces B: Biointerfaces 88: 96- 201 doi:10.1016/ j.colsurfb.2011.06.031

11.Xiangqian $\mathrm{Li}$, Huizhong $\mathrm{Xu}$, Zhe-Sheng Chen, and Guofang Chen (2011). Biosynthesis of nanoparticles by microorganisms and their applications. Journal of Nanomaterials, Volume 2011, Article ID 270974, 16 pages doi:10.1155/2011/270974 